\title{
Prospects of cancer biotherapy
}

\author{
Xishan Hao \\ Co-Editor-in-Chief, Drug Discoveries \& Therapeutics
}

\author{
Xishan Hao M.D., FACS \\ Member, Chinese Academy of Engineering; \\ President and Professor, Tianjin Medical University; \\ President and Professor, Tianjin Medical University Cancer \\ Institute and Hospital; President, China Anti-Cancer Association
}

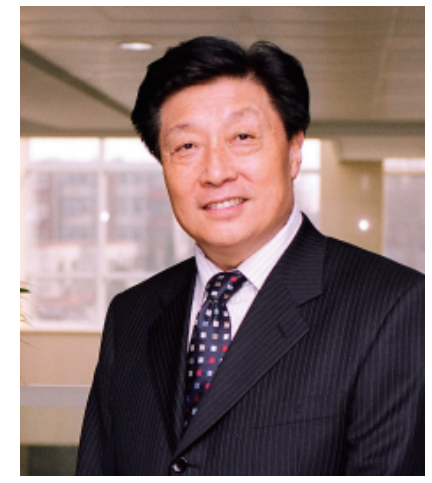

Thus far, more than 10 molecule targeted drugs applied in solid tumor treatment and several such drugs employed in hematological malignancy remedies have been approved for cancer therapy (8).

Research and development of novel molecule targeted drugs is extensively carried out nowadays. Drugs with startling efficacy have continuously sprung up in the recent two years. As the survival time of patients is the gold standard for evaluating drug efficacy, the value of molecule targeted therapy has two components. On the one side, the five-year survival or cure rate of cancer patients may be raised by using the targeted drugs to reduce tumor cell differentiation or in combination with conventional surgical treatment, radiotherapy and chemotherapy. This category of drugs includes: all-trans retinoic acid (for acute promyelocytic leukemia), trastuzumab (for breast carcinoma), rituximab (for lymphoma) and imatinib (for gastrointestinal stromal tumors and chronic myeloid leukemia). On the other side, the targeted therapy may delay tumor progression, thus improving life quality and prolonging the lifetime of patients. Along with the advancing research, the clinical value of molecule targeted therapy will be enriched and expanded.

Gene therapy: Gene therapy is a biomedical technique that overcomes genetic defects or exerts therapeutic effects via transduction of normal or therapeutic genes into targeted cells in certain ways, thus achieving the purpose of treatment of diseases (9). The limitations of conventional cancer therapies such as surgical treatment, radiotherapy and chemotherapy have prompted searching for novel treatments. With the indepth understanding of molecular mechanisms involving in tumor development and progression, it was realized that tumors are genopathies from the viewpoint of genetics. Correcting genetic defects may offer a new hope for cancer management.

There are two key issues that should be resolved in gene therapy. First comes the screening of the potential 
genes and subsequent is the control of the safety of vectors. Based on the therapeutic strategies, the targeted genes could be proto-oncogenes or antisense nucleic acid of genes that encode tumor cell autocrine growth factors and their receptors, tumor suppressor genes, immune regulatory factor genes, anti-angiogenesis factor genes, tumor cell suicide genes, antitumor antibody genes, etc. According to the types of vectors, gene therapy has experienced a developing process from naked DNA, nonviral gene delivery systems, to viral gene delivery systems. Up to the present, clinical treatment schemes that use adenovirus as a gene transfer vector are most widely used in gene therapy.

The number of clinical gene therapy programs provided by the journal $J$ Gene Med reached 1,537 in May 2009 , in which the number of gene therapies for cancer treatment is 993, making up about 2/3 of all projects (10). However, such therapies are mainly in phase I/II clinical trials so far.

Cell therapy: Tumor cellular immunotherapy is a therapeutic method that utilizes biotechnologies and biological agents to separate in vitro, activate and reintroduce tumor specific or non-specific effector cells, either autologous or allogenic, into cancer patients (11). Compared with traditional tumor therapies, it mainly focuses on improving the status of low cellular immune function and strengthening the host's antitumor immune response.

Adoptive cellular immunotherapy could be classified into two categories based on the antigen-specificity of the infused cells: non-specific cellular immunotherapy in which the infused cells include lymphokine activated killer cells (LAK), cytokine induced killer cells (CIK) and dendritic cells (DC), and specific cellular immunotherapy in which the infused cells mainly comprise cytotoxic $\mathrm{T}$ cells (CTL) and helper T cells (Th).

Up to now, many clinical studies of non-specific adoptive cellular immunotherapy have been performed and achieved some prospective results (12). Sipuleucel-T (Provenge), the first therapeutic cancer vaccine, was approved to treat advanced prostate cancer by the Food and Drug Administration (FDA) on April 29, 2010. The successful development of this drug which took over 20 years opened a new era of cancer immunotherapy. In addition, several phase I/II clinical studies of adoptive cellular immunotherapy based on CIK and DC have attained preliminary results in treatment of metastatic melanoma, liver cancer, metastatic renal cancer, and gastric cancer (13-15).

Specific adoptive cellular immunotherapy based on CTL has been the focus in immunotherapy of solid tumors such as melanoma, gastric cancer, colorectal cancer and liver cancer. Several phase I studies of such therapy have been accomplished (12). However, operative treatment schemes that can be applied in the clinic for cancer treatment are rare so far because of the low inducing efficiency of CTL in vitro, the complicated operation, and the long period and quantity limitation of amplification that often defeat the desired results.

Outlook: Biotherapy will be a main direction in cancer therapy in the future. In order to further increase its therapeutic effect in cancer treatment, efforts should be directed to the following aspects. First is the development of novel approaches of biotherapy to enhance the efficacy. Second is deeply and objectively evaluating the therapeutic effect of biotherapy through making randomized control studies in a large number of patients. Third, since biotherapy is largely adjuvant therapy and demonstrated to have a synergistic effect with conventional radiotherapy or chemotherapy, how to combine biotherapy with these traditional methods to achieve a better therapeutic effect in individualized cancer treatment should be explored in the future. Last, due to large individual differences in the efficacy of biotherapy, searching for biomarkers that could validly predict treatment outcome plays an important role in its clinical application. With the advancement of biotechnology, the means and efficacy of biotherapy will be improved constantly, which may make biotherapy occupy an increasingly important position in a comprehensive cancer treatment system.

\section{References}

1. Oldham RK. Biologicals and biological response modifiers: Fourth modality of cancer treatment. Cancer Treat Rep. 1984; 68:221-232.

2. Principles of Cancer Biotherapy. 5 ed., Springer Dordrecht Heidelberg London New York, 2009.

3. Song P, Tang W, Tamura S, Hasegawa K, Sugawara Y, Dong J, Kokudo N. The management of hepatocellular carcinoma in Asia: A guideline combining quantitative and qualitative evaluation. BioSci Trends. 2010; 4:283-287.

4. Hao XS. Biological therapy: New hope for cancer treatment. Chinese Medicinal Biotechnology. 2008; 3:401.

5. Barros Costa RL. Targeted therapy: Comprehensive review. Am J Hosp Palliat Care. 2009; 26:137-146.

6. Gao JJ, Xue X, Qu XJ, Tang W. c-Met: A potential therapeutic target for hepatocellular carcinoma. Drug Discov Ther. 2011; 5:2-11.

7. Phay JE, Shah MH. Targeting RET receptor tyrosine kinase activation in cancer. Clin Cancer Res. 2010; 16:5936-5941.

8. U.S. Food and Drug Administration. http://www.fda.gov (accessed April 2011).

9. Zhao Y, Lam DH, Yang J, Lin J, Tham CK, Ng WH, Wang S. Targeted suicide gene therapy for glioma using human embryonic stem cell-derived neural stem cells genetically modified by baculoviral vectors. Gene Ther. 2011.

10. In this issue. Gene therapy. J Gene Med. 2009; 11:1075-1076.

11. Chow KK, Gottschalk S. Cellular immunotherapy for highgrade glioma. Immunotherapy. 2011; 3:423-434.

12. U.S. National Institutes of Health. http://clinicaltrials.gov (accessed April 2011).

13. Hui D, Qiang L, Jian W, Ti Z, Da-Lu K. A randomized, controlled trial of postoperative adjuvant cytokine-induced killer cells immunotherapy after radical resection of hepatocellular carcinoma. Dig Liver Dis. 2009; 41:36-41.

14. Su X, Zhang L, Jin L, Ye J, Guan Z, Chen R, Guo T. Immunotherapy with cytokine-induced killer cells in metastatic renal cell carcinoma. Cancer Biother Radiopharm. 2010; 25:465-470.

15. Jiang JT, Shen YP, Wu CP, Zhu YB, Wei WX, Chen LJ, Zheng X, Sun J, Lu BF, Zhang XG. Increasing the frequency of CIK cells adoptive immunotherapy may decrease risk of death in gastric cancer patients. World J Gastroenterol. 2010; 16:6155-6162. 